\title{
miR-219 inhibits the proliferation, migration and invasion of medulloblastoma cells by targeting CD164
}

\author{
JIA-ANG SHI $^{1 *}$, DA-LIN LU ${ }^{2 *}$, XUAN HUANG ${ }^{3}$ and WEI TAN ${ }^{2}$ \\ ${ }^{1}$ Department of Psychiatry, West China Hospital, Sichuan University, Chengdu, Sichuan 610041; \\ ${ }^{2}$ Tianyou Hospital Affiliated to Wuhan University of Science and Technology, Wuhan, Hubei 430081; \\ ${ }^{3}$ Medical College, Nanchang University, Nanchang, Jiangxi 330000, P.R. China
}

Received November 28, 2013; Accepted April 1, 2014

DOI: $10.3892 /$ ijmm.2014.1749

\begin{abstract}
It is known that microRNA-219 (miR-219) expression is downregulated in medulloblastoma. In the present study, we investigated the expression, targets and functional effects of miR-219 in D283-MED medulloblastoma cells. We first demonstrated that miR-219 not only inhibits proliferation, but also suppresses the invasion and migration of D283-MED cells. Moreover, the knockdown of miR-219 promoted the proliferation, migration and invasion of the D283-MED cells. Secondly, we predicted that miR-219 targets the 3' untranslated region (3'UTR) of CD164 and orthodenticle homeobox 2 (OTX2) and then confirmed that it significantly downregulated the protein expression of CD164 and OTX2 in D283-MED cells. Finally, we demonstrated that the proliferation, invasion and migration of D283-MED cells were promoted by theectopic expression of CD164. These results indicate that miR-219 suppresses the proliferation, migration and invasion of medulloblastoma cells by targeting CD164. The results also suggest that miR-219 may serve as a potential therapeutic agent for medulloblastoma.
\end{abstract}

\section{Introduction}

microRNAs (miRNAs or miRs) have emerged as one of the important regulators of the interaction network that controls various cellular processes. They are short non-coding RNAs which regulate target mRNAs by binding mostly in the

Correspondence to: Professor Wei Tan, Tianyou Hospital Affiliated to Wuhan University of Science and Technology, Wuhan, Hubei 430081, P.R. China

E-mail: tanwei63317@163.com

Dr Xuan Huang, Medical College, Nanchang University, Nanchang, Jiangxi 330000, P.R. China

E-mail: xuanhuang0403@gmail.com

${ }^{*}$ Contributed equally

Key words: medulloblastoma, microRNA-219, CD164, OTX2
3' untranslated region ( 3 'UTR), leading to either translational repression or degradation of the target. The aberrant expression of miRNAs has been reported in multiple human cancer types. miRNAs are known to play an oncogenic or tumor suppressor role and they have been shown to play key roles in cell survival, proliferation, apoptosis, migration and invasion; miRNAs also have various other characteristics which become altered in human cancers $(1,2)$.

Medulloblastoma is a malignant tumor of the cerebellum. The median age at diagnosis is 5 years, with the age range extending into young adulthood. Primary management consists of surgical resection followed by radiation therapy and chemotherapy. Current therapies have serious short-term and long-term adverse effects, including post-operative mutism, neurocognitive deficits, endocrinopathies, sterility and the risk of secondary high-grade glioma or meningioma (3). Patients with recurrent disease after primary therapy have a particularly poor prognosis, with a median survival of $<6$ months; the 2-year survival rate among these patients is approximately $9 \%$ (4). Thus, this necessitates the indepth elucidation of the regulation of pathways for the better understanding of the disease, which may lead to the development of personalized, more targeted therapies.

CD164 (endolyn) is a sialomucin, which has been shown to play roles in regulating the proliferation, adhesion and differentiation of hematopoietic stem cells (5). In addition, CD164 is highly expressed in colon cancer sites, and it promotes colon cancer cell proliferation and metastasis both in vitro and in vivo (6). It has also been suggested that CD164 plays a key role in prostate cancer metastasis (7). The reduction of the CD164 expression level has become a focus of research in colon cancer. However, to the best of our knowledge, its role in medulloblastoma has not been reported to date. Moreover, orthodenticle homeobox 2 (OTX2) is a known oncogene for medulloblastoma, and its aberrant overexpression has been shown to correlate with a poorer survival in patients with medulloblastoma (8).

In this study, we first demonstrate that miR-219 not only inhibits proliferation, but also suppresses the invasion and migration of D283-MED medulloblastoma cells. Moreover, the knockdown of miR-219 promotes the proliferation, migration and invasion of D283-MED cells. Secondly, we predicted that miR-219 targets the 3'UTR of CD164 and OTX2 and then confirmed that it significantly downregulated the protein 
expression of CD164 and OTX2 in D283-MED cells. Finally, we demonstrated that the proliferation, invasion and migration of D283-MED cells were promoted by the ectopic expression of CD164.

\section{Materials and methods}

Cell line and transfection. The D283-MED medulloblastoma cell line was obtained from the American Type Culture Collection (ATCC; Manassas, VA, USA). D283-MED cells were cultured in Improved-MEM (Zn Option) supplemented with $10 \% \mathrm{FBS}, 2 \mathrm{mmol} / \mathrm{l}$ L-glutamine, $2 \mathrm{mmol} / 1$ sodiumpyruvate, $100 \mathrm{U} / \mathrm{ml}$ penicillin and $100 \mathrm{mg} / \mathrm{ml}$ streptomycin. The cells were maintained in a humidified atmosphere containing $5 \% \mathrm{CO}_{2}$ at $37^{\circ} \mathrm{C}$.

Transfection was performed using Lipofectamine 2000 reagent (Invitrogen, Carlsbad, CA, USA) according to the manufacturer's instructions. miR-219 mimics, mimic negative controls (m-NCs), miR-219 inhibitor and inhibitor negative controls (i-NCs) were purchased from RiboBio Co., Ltd. (Guangzhou, China).

A final concentration of $50 \mathrm{nM}$ of mimics or $100 \mathrm{nM}$ of inhibitor and their respective negative controls were used for each transfection in MTT assay, BrdU incorporation, invasion and migration expreriments. CD164 expressiion plasmids and empty vector (pcDNA3.1) were purchased from the National RNAi Core Facility in Academic Sinica, Taipei, Taiwan. The concentration of the CD164 expression plasmids or the empty vector (pcDNA3.1) used for each transfection was $10 \mu \mathrm{g}$.

miRNA detection. Total RNA from the cultured cells, with efficient recovery of small RNAs, was isolated using the mirVana miRNA Isolation kit (Ambion, Austin, TX, USA). The detection of the mature form of miRNAs was performed using the mirVana qRT-PCR miRNA Detection kit and qRT-PCR Primer Sets, according to the manufacturer's instructions (Ambion). The U6 small nuclear RNA was used as an internal control.

Examination of cell proliferation by MTT assay. The examination of cell proliferation by MTT assay was performed as described in a previous study (9). The effects of miR-219 on cell proliferation were assessed by 3-(4, 5-dimethylthiazol2-yl)-2, 5-diphenyltetrazolium (MTT) assay (Sigma, St. Louis, MO, USA) assay. The absorbance was directly proportional to the number of surviving cells. The inhibition rate was calculated as follows: inhibition rate $=(1-\mathrm{A}$ experiment $/ \mathrm{A}$ control) $\mathrm{x} 100 \%$.

Analysis of cell proliferation by BrdU incorporation. Cell proliferation was assessed using a colorimetric BrdU cell proliferation kit according to the manufacturer's instructions (Cat. no. 11647229001; Roche, Indianapolis, IN, USA). The transfected cells were labeled with BrdU for 3 to $4 \mathrm{~h}$. The genomic DNA was fixed and denatured, followed by incubation with peroxidase-conjugated anti-BrdU antibody for $90 \mathrm{~min}$. A substrate for the conjugated peroxidase was then added and the reaction product was quantified by measuring the absorbance. The results were then normalized by the number of total viable cells.
Table I. Antibodies used in western blot analysis.

\begin{tabular}{ll}
\hline Primary antibody & Secondary antibody \\
\hline Anti-CD164 (1:500; Abcam) & Anti-rabbit secondary \\
Anti-OTX2 (1:500; Abcam) & antibodies (Li-COR) \\
Anti-ISL1 (1:500; Abcam) & \\
Anti-EYA1 (1:500; Abcam) & \\
Anti-RECK (1:500; Abcam) & \\
Anti-UBE2N (1:500; Abcam) & \\
Anti- $\beta$-actin (1:500; Abcam) & \\
\hline
\end{tabular}

Migration and invasion assay. For Transwell migration assays, $2.5 \times 10^{4}$ to $5 \times 10^{4}$ cells were plated in the top chamber with the non-coated membrane (24-well insert; pore size, $8 \mathrm{~mm}$; BD Biosciences, San Jose, CA, USA). For invasion assays, $1.25 \times 10^{5}$ cells were plated in the top chamber with Matrigel-coated membrane (24-well insert; pore size, $8 \mathrm{~mm}$; BD Biosciences). For both assays, the cells were plated in medium without serum, and medium supplemented with serum was used as a chemo-attractant in the lower chamber. The cells were incubated for $24 \mathrm{~h}$ and cells that did not migrate or invade through the pores were removed by a cotton swab. Cells on the lower surface of the membrane were stained with the Diff-Quick Stain Set (Dade Behring, Inc., Newark, DE, USA) and counted.

Reverse transcription-polymerase chain reaction. Total RNA was isolated from the cells using TRIzol reagent (Invitrogen). cDNA was synthesized from $1 \mu \mathrm{g}$ of total RNA in a $20 \mu \mathrm{l}$ reverse transcription (RT) system followed by PCR amplification in a 50- $\mu$ l PCR system performed using an RT-PCR kit (Promega, Madison, WI, USA). The housekeeping gene, glyceraldehyde-3-phosphate dehydrogenase (GAPDH), was used as an RNA loading control. The PCR primer sequences were as follows: CD164 forward, 5'-GTGCTGTCCGCGG ACAAGAAC-3' and reverse, 5'-TGTGAACAATAGCTCT CATC-3'; OTX2 forward, 5'-TCTTATCTAAAGCAACCG CCTTACGCAGTC-3' and reverse, 5'-GCACCCTGGATTCT GGCAAGTTGATTTTCA-3'; and GAPDH forward, 5'-TGT CATCAACGGGAAGCCCA-3' and reverse, 5'-TTGTCAT GGATGACCTTGGC-3'.

PCR was conducted according to manufacturer's instructions and the PCR products were analyzed by agarose gel electrophoresis. Gels were photographed and the densities of the bands were determined using a computerized image analysis system (Alpha Innotech, San Leandro, CA, USA). The area of each band was calculated as the integrated density value (IDV). Mean values were calculated from 3 separate experiments. The IDV ratios of CD164 to GAPDH and OTX2 to GAPDH were calculated for each sample.

Western blot analysis. Western blot analysis was performed as previously described (9). The antibodies used are listed in Table I. Briefly, following incubation with the primary antibody (Table I) overnight at $4^{\circ} \mathrm{C}$, IRDye ${ }^{\mathrm{TM}}-800$-conjugated antirabbit secondary antibodies (Li-COR Biosciences, Lincoln, NE, USA) were used for $30 \mathrm{~min}$ at room temperature. The 
A

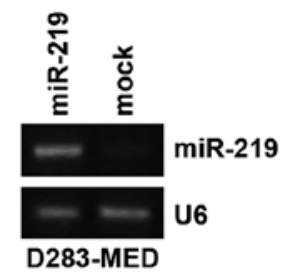

B

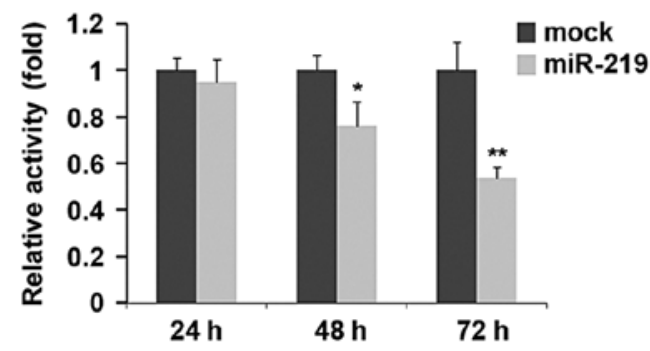

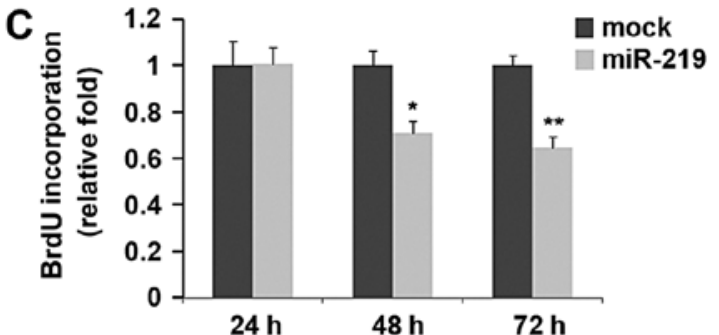

D

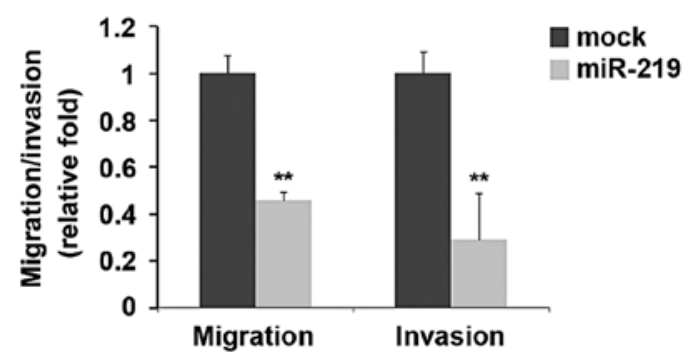

Figure 1. microRNA-219 (miR-219) inhibits the proliferation, migration and invasion of D283-MED cells. (A) RT-PCR of miR-219 in D283-MED cells infected with the miR-219 mimics or mimic negative controls (m-NCs; mock). Forty-eight hours after transfection, the cells were harvested for RT-PCR, n=3. (B) D283-MED cells were transfected with miR-219 mimics or m-NCs (mock) and then cell viability was measured at the indicated time points by MTT assay, $\mathrm{n}=3$. (C) D283-MED cells were transfected with miR-219 mimics or m-NCs (mock) and then BrdU incorporation assay was performed at the indicated time points, $n=3$. (D) Invasion and migration assays using D283-MED cells transfected with the miR-219 mimics or m-NCs (mock). Forty-eight hours after transfection, the cells were plated for invasion and migration assays, $n=3$.

specific proteins were visualized using an Odyssey ${ }^{\mathrm{TM}}$ Infrared Imaging System (Gene Company).

Microarray analysis. The preparation of RNA from the cells and analysis of the Affymetrix GeneChip microarray data (Affymetrix, Santa Clara, CA, USA) were carried out as previously described (10). Total RNA was prepared using TRIzol reagent (Invitrogen) according to the manufacturer's instructions. RNA was further purified using RNeasy columns and treatment with RNase-free DNase I (Qiagen, Chatsworth, CA, USA). Total RNA was used to generate cRNA, which was labeled with biotin as recommended by Affymetrix. cRNA was then hybridized to Affymetrix Hu95A GeneChips, which contain approximately 12,000 human oligonucleotide probe sets. After washing, the chips were scanned and analyzed using MicroArray Suite 5.0 (Affymetrix). The average intensities for each GeneChip were globally scaled to a target intensity of 150 . Further analysis was performed using GeneSpring software version 5.0.1 to obtain expression level information, fold change, and P-values for each gene relative to the control, as previously described (10). Genes with similar expression profiles were grouped together using hierarchical clustering and the resulting gene tree is shown. The magnitude of fold-induction or -repression for each miRNA (relative to the median of its expression across all experimental samples) is indicated by the colour bar. Data shown are based on 3 replicate studies.

Bioinformatics analysis. The analysis of potential microRNA target sites was carried out using 3 commonly used prediction algorithms: miRanda (http://www.microrna.org/), TargetScan (http://www.targetscan.org) and PicTar (http://pictar.bio.nyu. edu), as previously described (11).

\section{Results}

Overexpression of $\mathrm{miR}-219$ inhibits the proliferation, migration and invasion of D283-MED cells. In an attempt to identify the role of miR-219 in regulating the proliferation of medulloblastoma cells, D283-MED cells were transfected with miR-219 mimics. Following stable transfection, miR-219 expression was detected by RT-PCR and the proliferation rates of the D283-MED cells were determined by MTT assay. The results revealed that the exogenous miR-219 stably increased its expression in D283-MED cells (Fig. 1A). The overexpression of miR-219 significantly reduced the proliferation rate of the D283-MED cells after 48 and $72 \mathrm{~h}$ of transfection and the inhibition of cell proliferation was time-dependent (Fig. 1B). This was further revealed by BrdU incorporation assay, showing that transfection with miR-219 resulted in reduced DNA synthesis activity per viable cell in the D283-MED cells (Fig. 1C).

Given that miR-219 markedly inhibited D283-MED cell proliferation, we then sought to determine whether miR-219 has an effect on the migration and invasion of D283-MED cells. The cell migration and invasion assay of D283-MED cells revealed that the overexpression of miR-219 not only inhibited the migration of D283-MED cells, but also suppressed their invasion (Fig. 1D).

Knockdown of miR-219 promotes the proliferation, migration and invasion of D283-MED cells. To provide further evidence that miR-219 is involved in D283-MED cell proliferation, migration and invasion, we examined the effects of an inhibitor of miR-219. Following transfection, miR-219 expression was detected by RT-PCR and the proliferation rates 

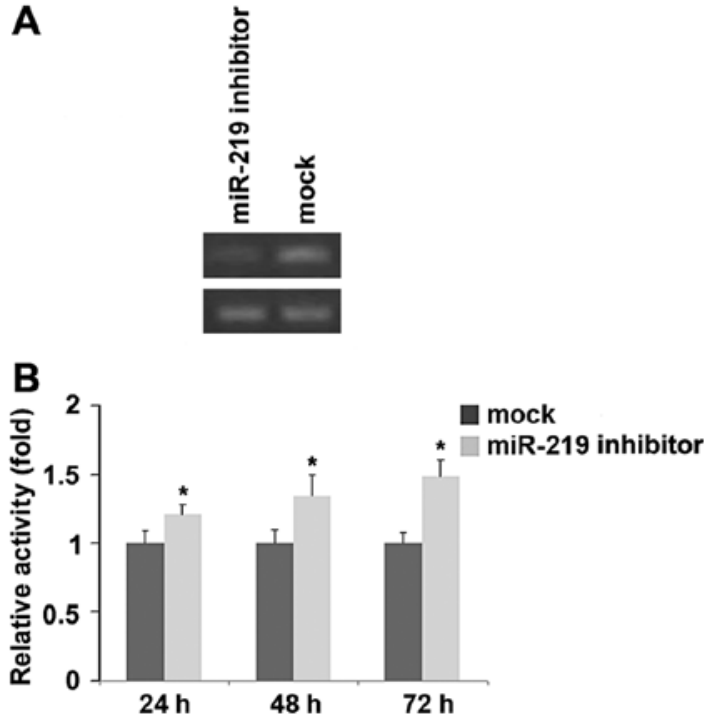

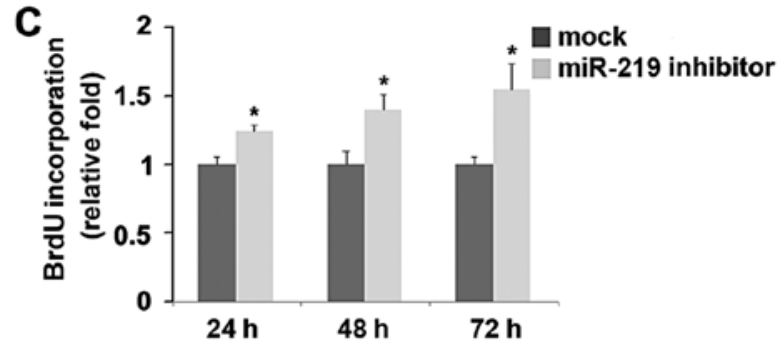

D

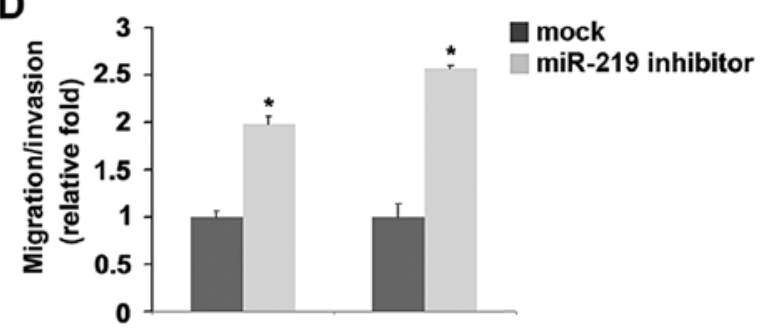

Figure 2. microRNA-219 (miR-219) inhibitor promotes the proliferation, migration and invasion of D283-MED cells. (A) RT-PCR of miR-219 in D283-MED cells infected with the miR-219 inhibitor or inhibitor negative controls (i-NCs) (mock). Forty-eight hours after transfection, the cells were harvested for RT-PCR, n=3. (B) D283-MED cells were transfected with miR-219 inhibitor or i-NCs (mock) and then cell viability was measured at the indicated time points by MTT assay, $n=3$. (C) D283-MED cells were transfected with miR-219 inhibitor or i-NCs (mock) and then BrdU incorporation assay was performed at the indicated time points, $n=3$. (D) Invasion and migration assays using D283-MED cells transfected with the miR-219 inhibitor or i-NCs (mock). Forty-eight hours after transfection, the cells were plated for invasion and migration assays, $\mathrm{n}=3$.

of the D283-MED cells were examined by MTT assay. The results revealed that miR-219 inhibitior decreased miR-219 expression in the D283-MED cells. In addition, the proliferation of D283-MED cells transfected with miR-219 inhibitor was found to be higher than that of the cells transfected with i-NCs (Fig. 2A and B). Consistent with the results of MTT assay, BrdU incorporation assay demonstrated that DNA synthesis was increased by miR-219 inhibitor in the cells (Fig. 2C). Finally, we found that miR-219 inhibitor also markedly increased the migration and invasion of the D283-MED cells (Fig. 2D). miR-219 inhibitors play an opposite role to miR-219 in regulating the proliferation, migration and invasion of D283-MED cells.

CD164 and OTX2 protein but not mRNA expression is suppressed by miR-219 in D283-MED cells. We then performed an analysis of potential microRNA target sites using 3 commonly used prediction algorithms, miRanda (http://www.microrna. org/), TargetScan (http://www.targetscan.org) and PicTar (http://pictar.bio.nyu.edu) (11). All 3 algorithms predicted the CD164, eyes absent homolog 1 (Drosophila) (EYA1), ISL LIM homeobox 1 (ISL1), lysosomal protein transmembrane 4 alpha (LAPTM4A), OTX2, reversion-inducing-cysteine-rich protein with kazal motifs (RECK), T, Brachyury homolog (mouse) (T), thyrotropin-releasing hormone degrading enzyme (TRHDE) and ubiquitin-conjugating enzyme E2N (UBE2N) genes to be targets of miR-219 (Fig. 3A).

In an attempt to further identify the role of miR-219 in regulating gene expression in medulloblastoma cells, the D283-MED cells were transfected with miR-219, and then western blot analysis was performed. Due to the lack of LAPTM4A, T and TRHDE antibodies, we only detected CD164, OTX2, ISL1, EYA1, RECK and UBE2N protein expression. We found that
miR-219 markedly inhibited the expression of 2 out of the 6 genes, CD164 and OXT2 in the D283-MED cells (Fig. 3B). A total of 2 miR-219 target sites (Fig. 3C) were found in the 3'UTR of CD164 and 1 target site (Fig. 3D) was found in the 3'UTR of OTX2 by miRanda, TargetScan and PicTar. Furthermore, RT-PCR revealed that the mRNA levels of CD164 and OTX2 were not significantly affected by miR-219 in the D283-MED cells (Fig. 3E and F).

Overexpression of CD164 promotes the proliferation, migration and invasion of D283-MED cells. We confirmed that miR-219 inhibited proliferation, migration and invasion, and reduced CD164 expression in D283-MED cells. We hypothesized that CD164 plays an opposite role to miR-219 in D283-MED cells. Firstly, the D283-MED cells were transfected with CD164 expression plasmids. Following transfection, CD164 expression was detected by western blot analysis and the results revealed that the CD164 expression plasmids markedly increased CD164 protein expression in the D283-MED cells (Fig. 4A). After 24, 48 and $72 \mathrm{~h}$ of stable transfection, the proliferation rates of the D283-MED cells were determined by MTT assay. The results revealed that the overexpression of CD164 markedly increased the proliferation rate of the D283-MED cells by approximately $25-150 \%$ and the promotion of cell growth was time-dependent (Fig. 4B). This was further revealed by BrdU incorporation assay, showing that transfection with CD164 resulted in increased DNA synthesis activity per viable cell and the promotion of cell growth was time-dependent in the D283-MED cells (Fig. 4C).

Given that CD164 markedly increased the proliferation of D283-MED cells, we then sought to determine whether CD164 has any effect on the migration and invasion of D283-MED cells. Of note, the results of cell migration and invasion assay 
A

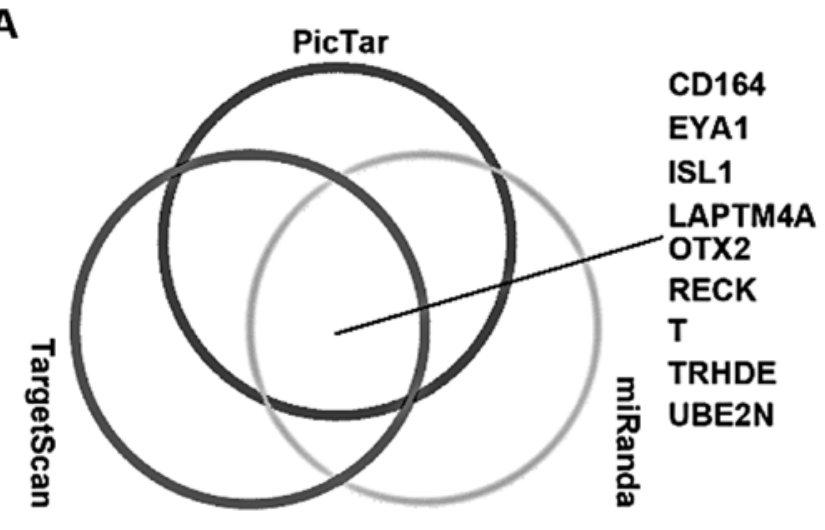

B
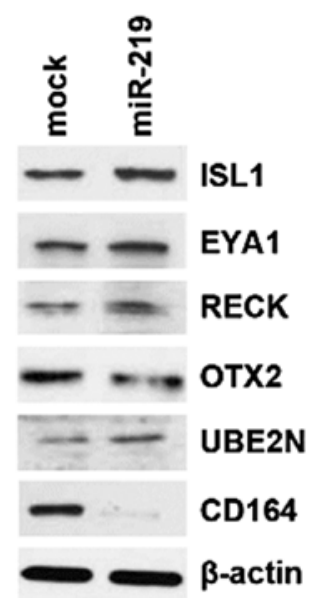

D

\section{C}

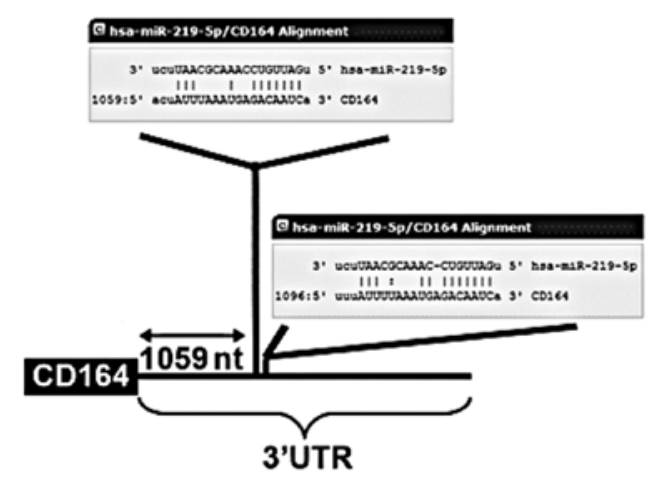

E

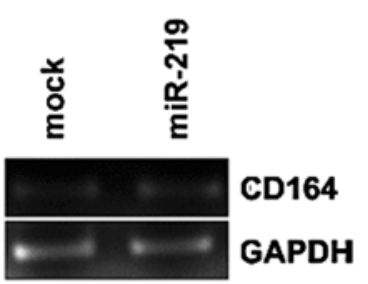

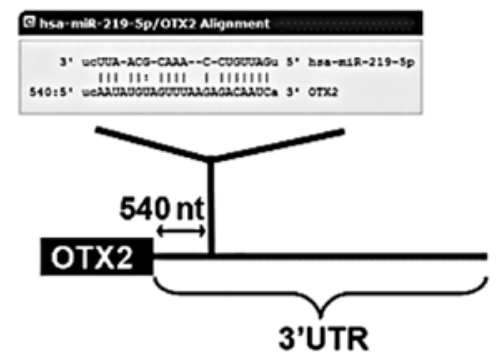

$\mathbf{F}$

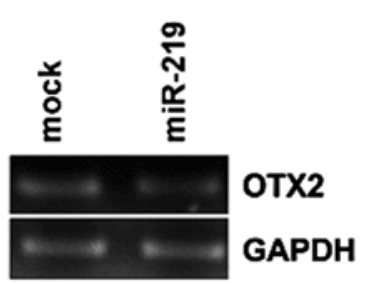

Figure 3. microRNA-219 (miR-219) reduces CD164 and OXT2 expression in D283-MED cells. (A) Diagram showing the predicted target genes of miR-219 from databases (miRanda, TargetScan and PicTar). (B) Western blot analysis results of ISL1, EYA1, RECK, OTX2, UBE2N and CD164 protein expression in D283-MED cells transfected with miR-219 mimics or mimic negative controls (m-NCs; mock) at $48 \mathrm{~h}$ after transfection, $\mathrm{n}=3$. (C) miRanda, TargetScan and PicTar predicted that miR-219 targets the 3'UTR of CD164. (D) miRanda, TargetScan and PicTar predicted that miR-219 targets the 3'UTR of OTX2. (E) Expression of miR-219 reduced CD164 protein expression, but not mRNA levels. D283-MED cells were transfected with miR-219 mimics or m-NCs and then subjected to RT-PCR. (F) Expression of miR-219 reduced OTX2 protein expression, but not mRNA levels. D283-MED cells were transfected with miR-219 mimics or m-NCs and then subjected to RT-PCR.

revealed that the overexpression of CD164 not only increased the migration of D283-MED cells, but also promoted their invasion (Fig. 4D).

CD164 regulates cell cycle-associated gene expression in D283-MED cells. To identify the cell cycle-associated genes that may be involved in the CD164-mediated regulation of the proliferation of D283-MED cells, we used Affymetrix GeneChip DNA microarrays to compare the gene expression profiles in the D283-MED cells transfected with CD164 expression plasmids or the empty vector.

RNA was isolated from the D283-MED cells transfected with CD164 expression plasmids or the empty vector (mock). Total RNA was used to generate cRNA, which was labeled with biotin as recommended by Affymetrix. cRNA was then hybridized to Affymetrix Hu95A GeneChips, which contain approximately 12,000 human oligonucleotide probe sets. Analysis of the significantly regulated genes, as described in Materials and methods, identified 99 genes which were regulated by $>2$-fold (i.e.,either upregulated or downregulated) by CD164 in the D283-MED cells. We identified 6 genes, c-myc, PCNA, CDK6, CK19, Ki67 and CDK1, that were commonly associated with cell cycle regulation (Fig. 5).

\section{Discussion}

The role of miRNAs in medulloblastoma has only recently been addressed and more recently, studies have implicated 
A

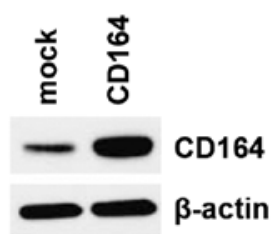

B

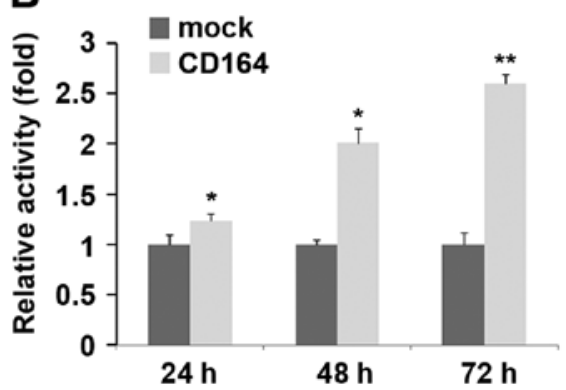

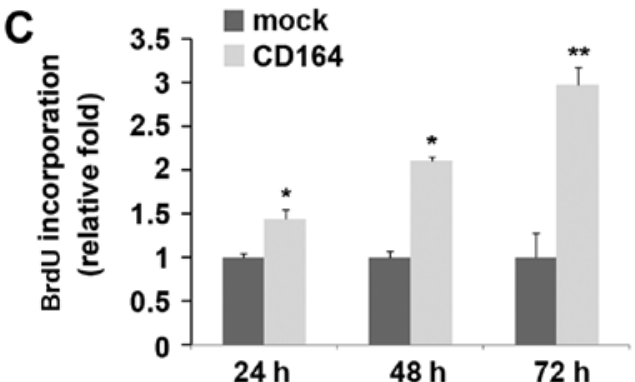

D

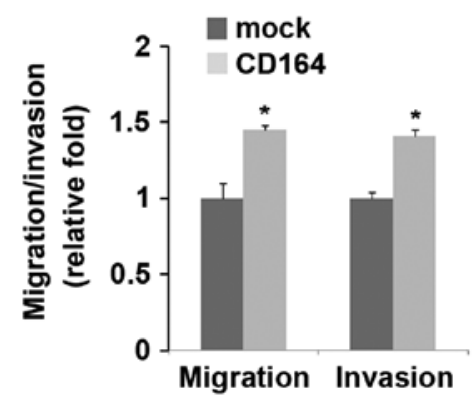

Figure 4. Proliferation, migration and invasion of D283-MED cells is promoted by CD164. (A) Western blot analysis of CD164 in D283-MED cells infected with CD164 expression plasmids or empry vector (mock). Forty-eight hours after transfection, the cells were harvested for western blot analysis, $\mathrm{n}=3$. (B) D283-MED cells were infected with CD164 expression plasmids or empty vector (mock) and then cell viability was measured at the indicated time points by MTT assay, $\mathrm{n}=3$. (C) D283-MED cells were infected with CD164 expression plasmids or empty vector (mock) and then BrdU incorporation assay was performed at the indicated time points, n=3. (D) Invasion and migration assays using D283-MED cells infected with CD164 expression plasmids or empty vector (mock). Forty-eight hours after transfection, the cells were plated for invasion and migration assays, $\mathrm{n}=3$.

\section{0}

3.7250051

4.98049

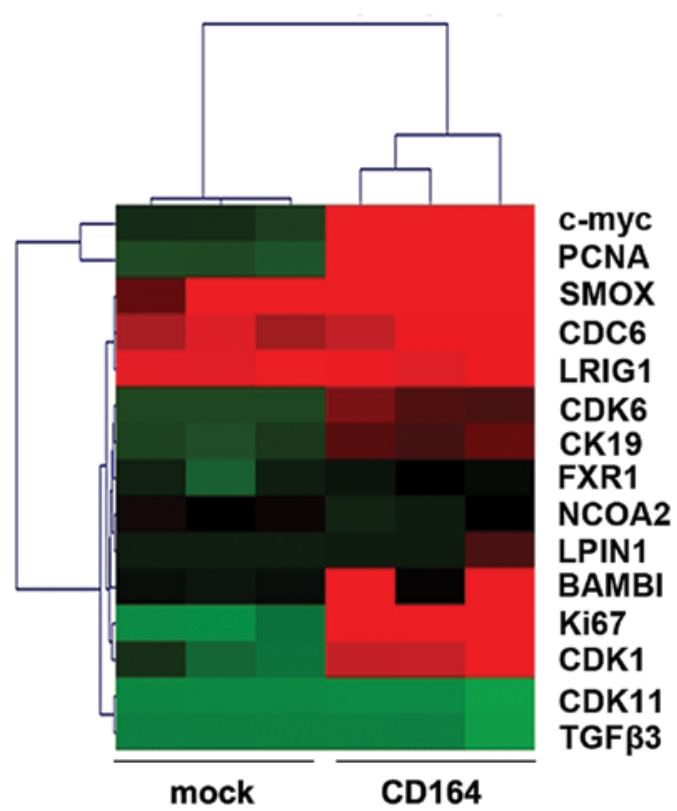

Figure 5. The contribution of CD164 to gene regulation identified by microarray analysis. Microarray analysis of D283-MED cells transfected with CD164 expression plasmids or empty vector (mock) was performed at $48 \mathrm{~h}$ after transfection. Genes with similar expression profiles were grouped together using hierarchical clustering and the resulting gene tree is shown. Color bar at the top indicates the magnitude of gene regulation, with green and red indicating repression and stimulation, respectively. Data shown are based on 3 replicate experiments.
miRNAs as important regulators of medulloblastoma cell survival, proliferation and migration. For example, miR-128a increases the intracellular reactive oxygen species (ROS) level by targeting Bmi-1 and inhibits medulloblastoma cell growth by promoting senescence (12); the pleiotropic effects of miR-183-96-182 converge to regulate the cell survival, proliferation and migration of medulloblastoma (13); miR-199b-5p can impair cancer stem cell growth through the negative regulation of HES1 in medulloblastoma (14); miR-182 promotes the leptomeningeal spread of non-sonic hedgehog-medulloblastoma (15); miR-218 acts as a tumor suppressor by targeting multiple cancer phenotype-associated genes in medulloblastoma (16); miR-21 suppression impedes medulloblastoma cell migration (17); the ectopic expression of miR-383 in medulloblastoma cells leads to the suppression of cell growth (18); miR-124 inhibits cell proliferation in medulloblastoma cells (19). Recent studies have reported that miR-219 is required for normal oligodendrocyte differentiation/myelination and inhibits hepatocellular carcinoma cell proliferation (20,21). Although the downregulation of miR-219 expression is known to be involved in the development of medulloblastoma, the functional role of miR-219 in medulloblastoma has not been previously described (22).

In this study, we report the novel regulation of proliferation, migration and invasion by miR-219 through the specific inhibition of CD164 in medulloblastoma cells. Our data are in line with those of previous studies, showing that miR-219 is downregulated in medulloblastoma samples, suggesting a tumor growth-inhibitory function (22), suggesting that the overexpression of miR-219 inhibits proliferation, migration 
and invasion and that the knockdown of miR-219 promotes the proliferation, migration and invasion of D283-MED cells. This further confirms that miR-219 is a tumor suppressor gene in medulloblastoma.

Further analysis of the possible mechanisms involved revealed that CD164 is a putative target of miR-219. We demonstrated that CD164 protein, but not mRNA expression was downregulated by miR-219 in the D283-MED cells. Consistent with the results of previous studies on CD164 in prostate and colon cancer, our results demonstrated that CD164 promoted the proliferation, migration and invasion of D283-MED cells $(4,6)$. In addition, the regulation of CD164 by miR-219 was further supported by the results that CD164 played an opposite role to miR-219 in regulating the proliferation, migration and invasion of medulloblastoma cells. OTX2 is known as an oncogene for medulloblastoma, and its aberrant overexpression has been shown to correlate with a poorer survival in patients with medulloblastoma (8). As prevoiusly demonstrated, the ectopic OTX2 expression enhanced the proliferation and tumorigenicity of immortalized primary cells, whereas OTX2 knockdown in medulloblastoma cells prolonged the survival of animals bearing tumor xenografts (8). In this study, we demonstrated that miR-219 inhibited OTX2 protein expression in D283-MED cells.

In conclusion, in this study, we describe the functional roles of miR-219 in D283-MED cells, and demonstrate that miR-219 decreases D238-MED cell proliferation, migration and invasion by downregulating CD164. It is more meaningful to demonstrate whether CD164 expression is downregulated in medulloblastoma and that miR-219 and CD164 are inversely expressed in medulloblastoma. Moreover, the modulation of miR-219 in in vivo studies using medulloblastoma models may shed light on the utility of the restoration of the expression of the tumor suppressor, miR-219, as a therapeutic modality for medulloblastoma.

\section{Acknowledgements}

This study was financially supported by grants from the National Natural Science Foundation of China (nos. 31071126, 31000343, 31171303, 31171297 and 31070694).

\section{References}

1. Esteller M: Non-coding RNAs in human disease. Nat Rev Genet 12: 861-874, 2011.

2. Esquela-Kerscher A and Slack FJ: Oncomirs - microRNAs with a role in cancer. Nat Rev Cancer 6: 259-269, 2006

3. Crawford JR, MacDonald TJ and Packer RJ: Medulloblastoma in childhood: new biological advances. Lancet Neurol 6: 1073-1085, 2007.

4. Zeltzer PM, Boyett JM, Finlay JL, et al: Metastasis stage, adjuvant treatment, and residual tumor are prognostic factors for medulloblastoma in children: conclusions from the Children's Cancer Group 921 randomized phase III study. J Clin Oncol 17: 832-845, 1999.

5. Doyonnas R, Yi-Hsin Chan J, Butler LH, et al: CD164 monoclonal antibodies that block hemopoietic progenitor cell adhesion and proliferation interact with the first mucin domain of the CD164 receptor. J Immunol 165: 840-851, 2000.
6. Tang J, Zhang L, She X, et al: Inhibiting CD164 expression in colon cancer cell line HCT116 leads to reduced cancer cell proliferation, mobility, and metastasis in vitro and in vivo. Cancer Invest 30: 380-389, 2012.

7. Havens AM, Jung Y, Sun YX, et al: The role of sialomucin CD164 (MGC-24v or endolyn) in prostate cancer metastasis. BMC Cancer 6: 195, 2006.

8. Adamson DC, Shi Q, Wortham M, et al: OTX2 is critical for the maintenance and progression of Shh-independent medulloblastomas. Cancer Res 70: 181-191, 2010.

9. Luo XG, Zou JN, Wang SZ, Zhang TC and Xi T: Novobiocin decreases SMYD3 expression and inhibits the migration of MDA-MB-231 human breast cancer cells. IUBMB Life 62: 194-199, 2010.

10. Frasor J, Danes JM, Komm B, Chang KC, Lyttle CR and Katzenellenbogen BS: Profiling of estrogen up- and downregulated gene expression in human breast cancer cells: insights into gene networks and pathways underlying estrogenic control of proliferation and cell phenotype. Endocrinology 144: 4562-4574, 2003.

11. Sethupathy P, Megraw M and Hatzigeorgiou AG: A guide through present computational approaches for the identification of mammalian microRNA targets. Nat Methods 3: 881-886, 2006.

12. Venkataraman S, Alimova I, Fan R, et al: MicroRNA 128a increases intracellular ROS level by targeting Bmi-1 and inhibits medulloblastoma cancer cell growth by promoting senescence. PLoS One 5: e10748, 2010.

13. Weeraratne SD, Amani V, Teider N, et al: Pleiotropic effects of miR-183 96 182 converge to regulate cell survival, proliferation and migration in medulloblastoma. Acta Neuropathol 123: 539-552, 2012.

14. Garzia L1, Andolfo I, Cusanelli E, et al: MicroRNA-199b-5p impairs cancer stem cells through negative regulation of HES1 in medulloblastoma. PLoS One 4: e4998, 2009.

15. Bai AH, Milde T, Remke M, Rolli CG, Hielscher T, Cho YJ, Kool M, Northcott PA, Jugold M, Bazhin AV, Eichmüller SB, Kulozik AE, Pscherer A, Benner A, Taylor MD, Pomeroy SL, Kemkemer R, Witt O, Korshunov A, Lichter P and Pfister SM: MicroRNA-182 promotes leptomeningeal spread of non-sonic hedgehog-medulloblastoma. Acta Neuropathol 123: 529-538, 2012 .

16. Venkataraman S, Birks DK, Balakrishnan I, Alimova I, Harris PS, Patel PR, Handler MH, Dubuc A, Taylor MD, Foreman NK and Vibhakar R: MicroRNA 218 acts as a tumor suppressor by targeting multiple cancer phenotype-associated genes in medulloblastoma. J Biol Chem 288: 1918-1928, 2013.

17. Grunder E, D'Ambrosio R, Fiaschetti G, Abela L, Arcaro A, Zuzak T, Ohgaki H,Lv SQ, Shalaby T and Grotzer M: MicroRNA-21 suppression impedes medulloblastoma cell migration. Eur J Cancer 47: 2479-2490, 2011

18. Li KK, Pang JC, Lau KM, Zhou L, Mao Y, Wang Y, Poon WS and $\mathrm{Ng} \mathrm{HK}$ : MiR-383 is downregulated in medulloblastoma and targets peroxiredoxin 3 (PRDX3). Brain Pathol 23: 413-425, 2013.

19. Li KK, Pang JC, Ching AK, Wong CK, Kong X, Wang Y, Zhou L, Chen $\mathrm{Z}$ and $\mathrm{Ng} \mathrm{HK}$ : miR-124 is frequently down-regulated in medulloblastoma and is a negative regulator of SLC16A1. Hum Pathol 40: 1234-1243, 2009.

20. Dugas JC, Cuellar TL, Scholze A, Ason B, Ibrahim A, Emery B, Zamanian JL, Foo LC, McManus MT and Barres BA: Dicerl and miR-219 are required for normal oligodendrocyte differentiation and myelination. Neuron 65: 597-611, 2010.

21. Huang N, Lin J, Ruan J, Su N, Qing R, Liu F, He B, Lv C, Zheng D and Luo R: MiR-219-5p inhibits hepatocellular carcinoma cell proliferation by targeting glypican-3. FEBS Lett 586: 884-891, 2012.

22. Ferretti E, De Smaele E, Po A, Di Marcotullio L, Tosi E, Espinola MS, Di Rocco C, Riccardi R, Giangaspero F, Farcomeni A, Nofroni I, Laneve P, Gioia U, Caffarelli E, Bozzoni I, Screpanti I and Gulino A: MicroRNA profiling in human MB. Int J Cancer 124: 568-577, 2009. 